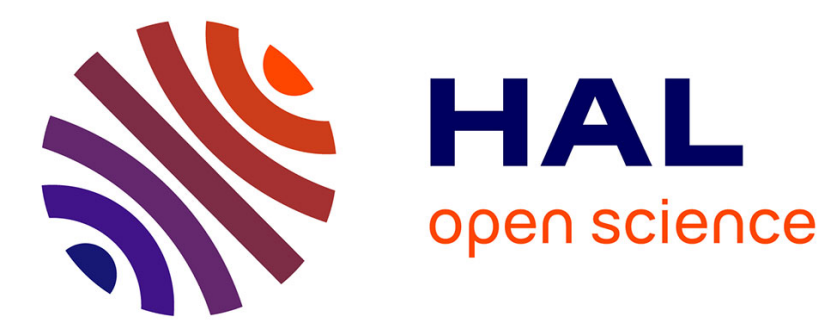

\title{
Remote Sensing Image Classification Using Attribute Filters Defined over the Tree of Shapes
}

Gabriele Cavallaro, Mauro Dalla Mura, Jon Atli Benediktsson, Antonio Plaza

\section{To cite this version:}

Gabriele Cavallaro, Mauro Dalla Mura, Jon Atli Benediktsson, Antonio Plaza. Remote Sensing Image Classification Using Attribute Filters Defined over the Tree of Shapes. IEEE Transactions on Geoscience and Remote Sensing, 2016, 54 (7), pp.3899-3911. 10.1109/TGRS.2016.2530690 . hal01272019

\section{HAL Id: hal-01272019 https://hal.science/hal-01272019}

Submitted on 10 Feb 2016

HAL is a multi-disciplinary open access archive for the deposit and dissemination of scientific research documents, whether they are published or not. The documents may come from teaching and research institutions in France or abroad, or from public or private research centers.
L'archive ouverte pluridisciplinaire HAL, est destinée au dépôt et à la diffusion de documents scientifiques de niveau recherche, publiés ou non, émanant des établissements d'enseignement et de recherche français ou étrangers, des laboratoires publics ou privés. 


\title{
Remote Sensing Image Classification Using Attribute Filters Defined over the Tree of Shapes
}

\author{
Gabriele Cavallaro, Student Member, IEEE, Mauro Dalla Mura, Member, IEEE, \\ Jón Atli Benediktsson, Fellow, IEEE, and Antonio Plaza, Fellow, IEEE
}

\begin{abstract}
Remotely sensed images with very high spatial resolution provide a detailed representation of the surveyed scene with a geometrical resolution that at the present can be up to 30 cm (WorldView-3). A set of powerful image processing operators have been defined in the mathematical morphology framework. Among those, connected operators (e.g., attribute filters) have proven their effectiveness in processing very high resolution images. Attribute filters are based on attributes which can be efficiently implemented on tree-based image representations. In this work, we considered the definition of min, max, direct and subtractive filter rules for the computation of attribute filters over the tree of shapes representation. We study their performance on the classification of remotely sensed images. We compare the classification results over the tree of shapes with the results obtained when the same rules are applied on the component trees. The random forest is used as a baseline classifier and the experiments are conducted using multispectral data sets acquired by QuickBird and IKONOS sensors over urban areas.
\end{abstract}

Index Terms-Remote sensing, Classification, Mathematical Morphology, Tree of Shapes.

\section{INTRODUCTION}

$\mathbf{R}$ EMOTE sensing instruments have been constantly improving their acquisition capabilities in terms of spatial resolution (e.g., WorldView-3: $0.3 \mathrm{~m}$ ) and spectral information (e.g., AVIRIS: 224 spectral channels). Very High Resolution (VHR) remotely sensed images provide a precise and detailed representation of a surveyed scene. The spatial information contained in these images can be fundamental for any application which requires a detailed analysis of the scene.

Such detailed automatic analysis and interpretation can be achieved by using mathematical morphology, a theory on morphological transformations, which has provided a set of very powerful tools for image processing. It originates from the seminal works of Matheron and Serra who worked

G. Cavallaro is with the Faculty of Electrical and Computer Engineering, University of Iceland, Reykjavik 101, Iceland (e-mail: cavallaro.gabriele@gmail.com).

M. Dalla Mura is with the GIPSA-lab, Grenoble Institute of Technology, Grenoble, France (e-mail: maurodallamura@gmail.com).

J. A. Benediktsson is with the Engineering Research Institute, University of Iceland, Reykjavik 107, Iceland (e-mail: benedikt@hi.is).

A. Plaza is with the Hyperspectral Computing Laboratory, Department of Technology of Computers and Communications, University of Extremadura, Caćeres 10071, Spain (e-mail: aplaza@unex.es).

This research was supported in part by the EU FP7 Theme Space project North State, by the program J. Verne 2014 under Project 31936TD, and by the INS2I JCJC 2015 under project "IDES". The authors are grateful to F. Pacifici for providing the Rome data set. on problems in petrography and mineralogy [1-3]. Due to their pioneering work, mathematical morphology [4] [5] has achieved the status of a fundamental set of toolkit in image processing and analysis and has provided solutions to many tasks in different domains, such as remote sensing [6][7], pattern recognition [8][9] and medical imaging [10][11]. Morphological operators perform image analysis mainly at the pixel-level and the region-level. For instance an image can be processed by considering the values in the neighborhood of each pixel. Such neighborhood is defined by the extent of a spatial mask commonly referred to as a structuring element (SE) [12]. By varying the size and the shape of the SE, the image is probed by different spatial windows leading to different results that can provide useful information about the shape and size of the objects present in the image.

Recently, region-based filtering tools [13][14] (called connected operators) have received significant attention. Contrary to classical morphological operators (i.e., based on SEs), connected operators are edge preserving since they act directly on the connected components where the image is constant, the so-called flat zones. As a consequence, the characteristics of the spatial features are not distorted since the connected operators can remove boundaries between flat zones but cannot add new boundaries or modify existing ones. Connected operators are capable of performing image transformations that can selectively suppress some details from the image and maintain unaffected structures that are relevant for the analysis. However, the spatial information belonging to VHR images present heterogeneous characteristics, thus a multilevel analysis is required in order to perform a complete modeling.

For this purpose, the authors in [15] introduced morphological profiles (MPs). MPs are a multiscale decomposition of a grayscale image composed by stacking the filtered images obtained by transforming the input image with a sequence of opening and closing by reconstruction filters based on SEs. The operators by reconstruction permit to filter an image by entirely preserving the geometry of those structures that are not erased from the scene. In [16], the MPs were applied for the first time in a remote sensing classification task. Nevertheless, there are limitations on the capabilities of modeling the spatial information. In particular, the profiles built by the filters based on SEs are not able to easily model other features than the size of the objects.

Breen and Jones originally proposed morphological attribute filters (AFs) [17], which have received increasing attention 


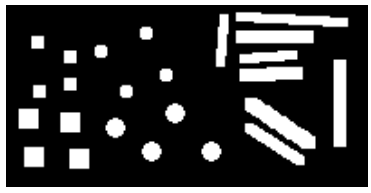

(a)

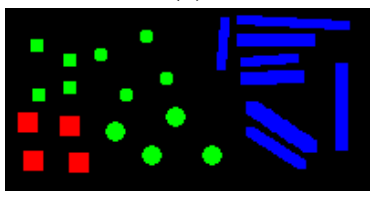

(c)

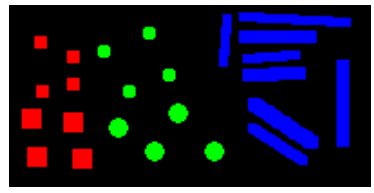

(b)

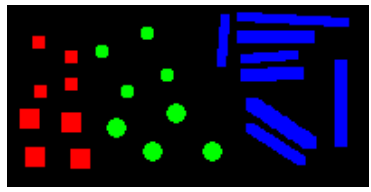

(d)
Fig. 1: Synthetic dataset: (a) binary image composed of three groups of foreground regions having different shapes (and different scales), (b) labeling of the pixels (4 classes), (c) classification with increasing attribute area and (d) classification with non increasing attribute moment of inertia.

due to the extended works presented in [18][19][20]. Attribute filters act by merging the connected components of a grayscale image according to a criterion evaluating one or more attributes computed on the image. They can overcome the main limitation of the MPs (i.e., they do not operate on SEs) due to their increased flexibility in defining operators based on attributes (i.e., the measures driving the type of filtering produced by the operator). Such an attribute can be related to the characteristics of the regions in the scene such as the area, perimeter, moment of inertia etc. The attribute filters can be efficiently implemented on the hierarchical representations of an image, such as the component trees (i.e., min-tree or max-tree [21] [22]) or the tree of shapes [23].

In such tree representations, each node corresponds to a region within the image. The filtering is not done on the image space, and it involves the creation of the tree structure, the analysis of each node by measuring a specific criterion, and the decision of whether to preserve or delete the node. The criteria are usually related to whether the value of a measure (i.e., attribute) fulfills a predefined condition. A criterion is said to be increasing if it is verified for a node and all the nodes nested in it. Examples of increasing criteria involve increasing attributes (such as area, volume, size of the bounding box, etc.). In contrast, non increasing attributes such as scale invariant measures (e.g., homogeneity, shape descriptors, orientation, etc.), lead to non increasing criteria, which means that the value of the attribute is not always greater for the ancestors of a node. The use of non-increasing attributes is sometimes necessary as shown in Fig. 1. In this example the objective is to perform a classification of the scene in which the discriminant feature is the shape of the regions. Thus, the objects in the foreground belong to different thematic classes according to their shape. Considering the area (i.e., increasing attribute) of the region as feature for the classification (result in Fig. 1(c)) leads to misclassifications, since some of the squared and circlular objects have similar size. Conversely, a shape descriptor such as the moment of inertia (non-increasing attribute) is able to discriminate all the different classes Fig. 1(d). When non increasing attributes are considered, arbitrary filter rules have to be defined in order to generate the outputs of the filter. Several filtering approaches have been proposed in the literature such as $\min$ [13], $\max$ [24], viterbi [21] and direct [25]. The first three strategies belong to the class of pruning strategy (i.e., entire branches are removed), while direct is a non pruning strategy (i.e., isolated nodes might be deleted). The authors in [26] [27] later showed that the aforementioned filter rules may not offer the best possible strategy when filtering grayscale images with non increasing attributes. For instance, the regions that are not supposed to be deleted by the filter may disappear in the local background or be merged with adjacent regions. Consequently, they proposed the non pruning strategy subtractive, a new filter rule which can be efficiently used for shape decomposition. The abovementioned rules have been proposed for dealing with min-tree and max-tree.

In this work, we considered the definition min, max, direct and subtractive for the computation of attribute filters over the tree of shapes. The hierarchy between its nodes is not driven by an ordering criterion of their graylevels (i.e., mintree and max-tree), since the ordering follows the inclusion relationship of the regions. In such structure, the application of non pruning strategies is not straightforward. For instance, in the subtractive rule, the operation of updating the descendants over the tree of shapes can introduce new graylevels in the filtered images which were not present in the original image. We study the effect of the filtering rules by considering non increasing attributes, such as standard deviation and moment of inertia. We show that, according to the selected attributes and the filter rule, the characterization of the spatial information is performed differently. The sequential application of attribute filters over the component trees and the tree of shapes generates multi-level decompositions of the image which are called Attribute Profiles (APs) [28] and Self-Dual Attribute Profiles (SDAPs) [29], respectively. In this contribution we evaluate the classification accuracies obtained by applying APs and SDAPs generated by different filter rules. SDAPs already proved to be more effective than APs [29] for the increasing attribute area, since bright and dark regions are simultaneously processed. In [30], we have presented a preliminary comparison of the impact of different filter rules in the context of classification. Yet this comparison was performed on a single data set, and the description of those rules and their comparison were not complete. This paper aims at extending this analysis, by presenting a full and exhaustive comparison conducted using multispectral data sets acquired by QuickBird and IKONOS sensors over urban areas.

The remainder of the paper is structured as follows. Section II reviews morphological attribute filters computed on mintree and max-tree and describes the filtering strategies in the context of increasing and non increasing operators. In Section III, we compute attribute filters with pruning and non pruning rules over the tree of shapes. The use of morphological attribute profiles in the context of classification of remotely sensed images is reviewed in Section IV. The experimental analysis, which includes the description of the data sets, the setup and the results, is given in section V. Section VI concludes this work with some remarks and hints at future research directions. 


\section{ATtRIBUte FILTERS BASED ON MIN AND MAX TREE}

Attribute filters are morphological operators that perform a region processing since they act by merging regions of constant value, the so-called flat zones. Attribute filters can be efficiently implemented taking advantage of hierarchical representations of the image as a tree. In the following these representations will be introduced along with the implementation of the filtering techniques based on the min-tree and max-tree.

\section{A. Min and Max tree}

Let $f$ be a discrete two-dimensional grayscale image, then its spatial domain $E$ is a set of positions which map into a set of scalar values $V, f: E \rightarrow V$ with typically $E \subseteq \mathbb{Z}^{2}$ and $V \subseteq \mathbb{Z}$. A flat zone of $f$ is a region of connected pixels $\mathcal{C C}_{v}(f)$ (connected component) of the level set $H_{v}(f)=\{x \in$ $E, f(x)=v\}$, with $v \in V$. At each gray level, there may be multiple connected components $\mathcal{C C}_{v}^{k}(f)$, with $k$ being some index variable. A peak component can be defined as the $k t h$ connected component $\mathcal{C C}_{v}^{k}(f)$ of the lower $\mathcal{L}(f)$ and upper $\mathcal{U}(f)$ threshold sets as [31]:

$$
\begin{aligned}
& \mathcal{L}(f)=\{x \in E, f(x)<v\}, \\
& \mathcal{U}(f)=\{x \in E, f(x) \geq v\},
\end{aligned}
$$

There is an inclusion relationship between the peak components extracted by $\mathcal{L}(f)$ and $\mathcal{U}(f)$ which allows to associate a node of a tree $N_{v}^{k}(f)$ to the subset of $\mathcal{C C}_{v}^{k}(f)$ with a fixed graylevel $v$ and represent the image as an hierarchical structure. The min-tree and max-tree structures represent the components in $\mathcal{L}(f)$ and $\mathcal{U}(f)$ respectively with their inclusion relations. The min-tree models the inclusion of regions according to the ordering graylevel criterion $(\leq)$, thus the tree contains only the shapes that are darker than their neighborhood (i.e., the graylevel of each region is lower than their neighborhood graylevel). The root of the min-tree is the entire image domain at the greatest grayscale value, while the leaves are the regional minima. The max-tree is dual, and it contains only the regions that are brighter than the graylevel of their neighboring pixels. In this case the root is the whole image at the lowest graylevel and leaves are the regional maxima. Component trees are widely used for computing attribute filters [17] [27], pattern spectra [27] [32], and multi-scale decompositions [33]. In [34] a complete comparison of the different algorithms proposed in the literature (sequential and parallel) for their computation is detailed.

\section{B. Increasing and Non increasing operators}

Once the tree representation has been created, the filtering step analyzes each node by measuring a specific criterion and takes a decision on the elimination or preservation of the node. The simplification itself is governed by a criterion (e.g., a binary predicate $P$ ) that may involve simple notions such as size, contrast, or more complex ones such as texture, motion, or even criteria close to semantic notions, such as similarity to predefined shapes. Taking as a reference the predicate $P=\alpha\left(N_{v}^{k}(f)\right) \geq \lambda$, an attribute $\alpha$ is computed over each node $N_{v}^{k}(f)$ and if it does not satisfy the predicate different strategies as remove/preserve decisions can be used [27]. According to the type of criteria (e.g., predicate) and the property of the attribute, the resulting operator can be defined as increasing or non increasing.

In the context of a tree structure, this characteristic is related to the criterion assessed for each node. When the predicate is in the form $P=\alpha\left(N_{v}^{k}(f)\right) \geq \lambda$ or $P=\alpha\left(N_{v}^{k}(f)\right) \leq \lambda$ and the attribute is increasing (i.e., the attribute of a node can never be less than the values of its descendants in the tree), the operator is also increasing. In this case, as shown in Fig. 2(b), there is no problem in defining a level where the criterion is higher or lower than a given $\lambda$ threshold. Contrarily, when the attribute is not increasing (i.e. the attribute of a node can be less than the values of its descendants in the tree), any predicate leads to a non increasing operation. In particular, as shown in Fig. 2(c), the criterion sequence fluctuates around the $\lambda$ threshold and defining the set of nodes to remove is less straightforward.

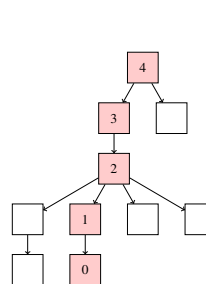

(a)

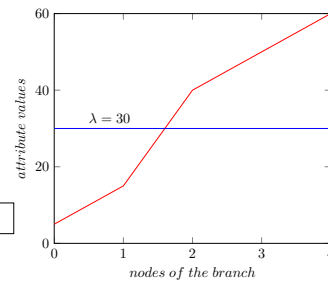

(b)

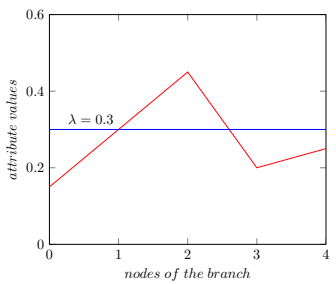

(c)
Fig. 2: Example of attribute values sequences for the nodes marked in red of the tree branch (a). Increasing attribute area (b), and non-increasing attribute moment of inertia (c). The attribute values sequence is marked are red, while the threshold imposed by the predicate $P$ is marked in blue.

\section{Connected operators: attribute filters}

In this work we use a specific category of connected operators called attribute filters. When attribute filters are applied over the tree representation of an image, the operator leads to a pruning of the tree by removing the nodes whose associated regions do not fulfill $P$.

Two general approaches might be used at this point: pruning and non pruning strategies. In the former, a single cut is made along each path from leaf to root, and all nodes leaf-side of the cut are collapsed onto the highest surviving ancestor. In the second class of rules, the simplification of the tree is not limited to the removal of entire branches but also isolated nodes might be removed along a root path. For example, when a node is deleted, the value of the pixels belonging to the node are updated to the value of its oldest surviving ancestor. Different approaches can be used for dealing with non increasing attributes. For example, the Viterbi algorithm [21] addresses the decision of removing or preserving a node as an optimization problem, while in [35] the authors apply the filtering on a graph whose nodes are weighted with an increasing order by the attribute.

In the following section, a description of pruning and non pruning strategies is provided by including the pseudo-code of 
the algorithms (a complete analysis can be found in [21] and [27]).

\section{Pruning strategy}

Pruning strategies consist in removing whole branches of the tree. They are simple to apply when the attribute is increasing since all nodes on which the criterion is not verified are organized in entire branches (i.e., if a node has to be removed, all of its descendants also have to be removed). When nodes of a branch are deleted, the level (i.e., graylevel) of their pixels are assigned to the level of the highest ancestor which satisfies the criterion.

The min rule prunes the branches from the leaves up to the last node that has to be removed. Therefore a node is removed if the predicate is false or if one of its ancestors is removed. The function Min rule (Algorithm 1) scans the tree starting from the root, and deletes a node when it does not satisfy the predicate or when its parent has to be removed.

The max rule cuts out the branches from the leaves up to the first node that has to be preserved. Thus, a node has to be removed if the predicate is false and all of its descendant nodes are deleted as well. In this case, the function Max rule (Algorithm 2) scans the tree starting from the leaves, and removes a node only when itself and its parent do not satisfy the predicate.

\section{E. Non pruning strategy}

A different type of image decomposition can be used in order to characterize heterogeneous regions and objects. The extraction of the pattern spectra [27] can be useful if the types of the details of interest are characterized by shape rather than size. This idea has been formalized as the so-called shape filters [26], and the operators which are anti-extensive and idempotent are not necessarily increasing. One example is the region perimeter: if a node $N_{0}^{k}(f)$ is included in region $N_{1}^{k}(f)$, no specific relation can be stated about their respective values. Non pruning strategies provide solutions for such cases where the simplification approach is not straightforward (i.e., the descendants of a node to be removed have not necessarily been removed).

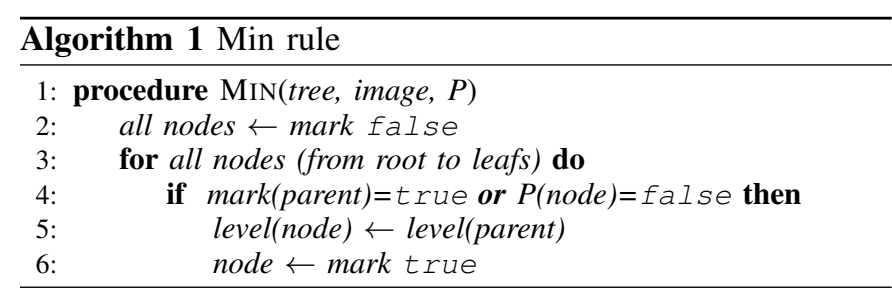

The direct rule consists simply in removing the nodes that do not fulfill the criterion. Thus, a node is removed if the predicate is false; its pixels are assigned to the graylevel of the highest ancestor which meets the criterion and its descendants are left unaffected (Algorithm 3). It has been proven in the literature that the direct rule is not the best strategy to deal with object enhancement and image decomposition based on shape [26]. The reason is that this strategy may lead to a loss of the contrast between the local background and

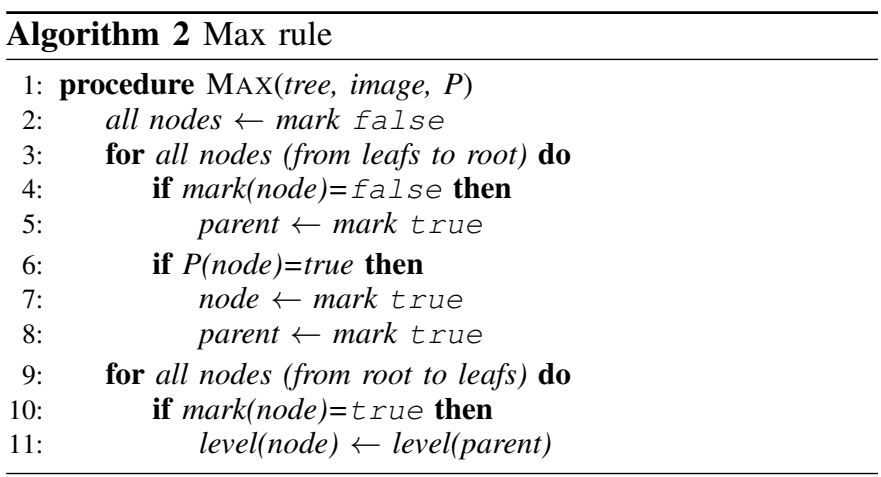

the surviving descendant regions. The consequence, which is shown in [26], is that the difference between the original image and the filtered image may contain structures that meet the aforementioned criterion (further details will be explained in the next section).

In order to solve the previous issues, the authors in [26] proposed the subtractive rule, as a simple and consistent approach for non increasing attributes. The first part of the algorithm performs the same as the direct rule, meaning that the nodes that do not satisfy the predicate are removed. Afterwards, the deletion of a node triggers a propagation process, which updates the graylevel of the surviving descendant nodes, so that the contrast with the local background remains invariant. Algorithm 4 shows that, for each descendant node, firstly, its value is set to the minimum, and then, by considering all graylevels in turn, a unit term is added every time the algorithm finds a connected component which satisfies the predicate and contains the considered node. A dual analysis can be done in the case of a min-tree, where first the maximum value is assigned and then a unit term is subtracted for each node which satisfies the previous requirements.
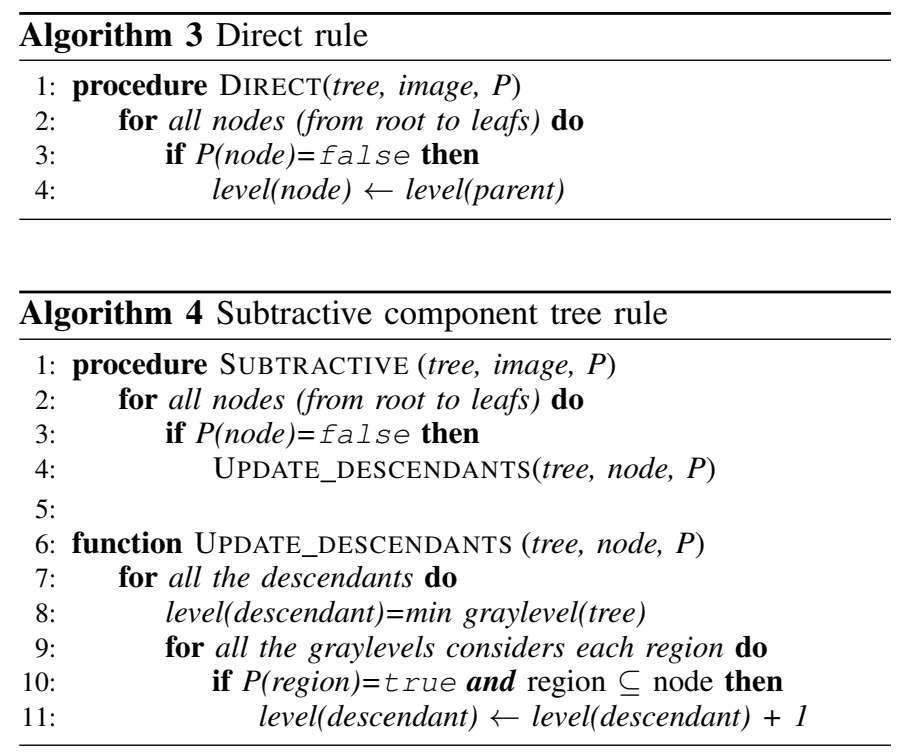


\section{ATTRIBUTE FILTERS BASED ON TREE OF SHAPES}

Pruning and non pruning strategies have been proposed for the min-tree and max-tree representations. In this section, we consider pruning and non pruning filter rules for the computation of attribute filters over the tree of shapes. We show that the definition of min, max, direct is equivalent to the case of component trees. We propose a different definition for the subtractive rule, since the principle that regulates the hierarchy of the nodes in the tree of shapes follows the inclusion of different structures.

\section{A. Tree of shapes}

A self-dual tree has been defined in [23], called the tree of shapes (also known as inclusion tree), that describes the image $f$ contents in a unique way; such a tree can be interpreted as the result of merging the min- and a max-tree of the same image. The tree of shapes is a morphological self-dual representation of the $\mathcal{C C}_{v}^{k}(f)$ within an image (i.e., zones enclosed by an isolevel line). It was firstly introduced by Monasse et al. [23], where the structure was computed with the Fast Level Line Transform (FLLT) algorithm: it first computes the pair of dual component trees and then obtains the tree of shapes by merging both trees. Afterwards, Caselles et al. [31] introduced the Fast Level Set Transform algorithm (FLST), which relies on a region-growing approach to decompose the image into shapes. An operation called saturation is applied to the connected components which gives flat regions obtained by progressively merging nested regions. Specifically, the algorithm extracts each branch of the tree starting from the leaves and growing them up to the root until only a single flat region is reached. Song et al. [36], proposed to retrieve the tree of shapes by building the tree of level lines and exploiting its interior of each level line. Recently Geraud et al. [37] proposed a new algorithm to compute the tree of shapes in order to reduce the computational complexity and overcome the restriction to only $2 \mathrm{D}$ images of the previous methods. The algorithm computes the tree of shapes with quasi-linear time complexity when data quantization is low (typically 12 bits or less) and it works for $\mathrm{nD}$ images. Moreover, Crozet et al. [38] presented the first parallel algorithm to compute the morphological tree of shapes based on the previous algorithm [37]. The tree of shapes is a more general representation of the image with respect to the min-tree and max-tree and it has many advantages.

An example of tree of shapes computation (i.e., region growing) is shown in Fig. 3 (f). The FLST algorithm extracts each branch of the tree starting from the leaves and growing them up to the root until only a single flat region is reached. It comprehends both the $\mathcal{L}(f)$ and $\mathcal{U}(f)$ sets, and intrinsically eliminates the redundancy of information contained in those sets. Min-tree and max-tree are representations of the image, and usually not all the connected components present in $\mathcal{L}(f)$ are also present in $\mathcal{U}(f)$ and vice versa. Since the tree of shapes is self-dual, it makes no assumption about the contrast of objects (either light object over dark background or the contrary). Finally, it encodes the spatial inclusion of $\mathcal{C C}_{h}^{k}(f)$

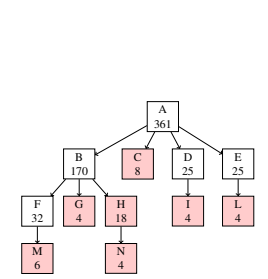

(a)

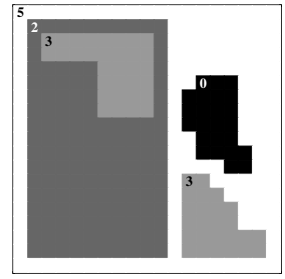

(b)

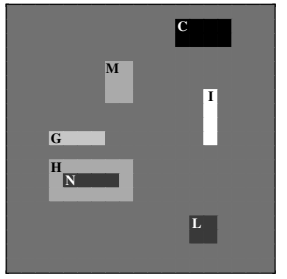

(c)
Fig. 4: Attribute filtering of the grayscale image $f$ in Fig. 3(a) using the area attribute with $P=\alpha(C C) \leq 20$. (a) Tree of shapes (nodes marked in red are removed). (b) Filtered image $\rho^{\mathrm{P}}(f)$ and (c) difference image $f-\rho^{P}(f)$.

in gray-level images so it is complementary to some other representations that focus on component (or region) adjacency.

\section{B. Increasing attributes}

When the attribute is increasing, the filtering is straightforward and it consists of removing whole branches of the tree. In particular, all the filtering rules (i.e., pruning and non pruning) lead to the same filtering result [30]. However, in [29] it was shown that the use of the tree of shapes as a structure representing the image allows simultaneously to access the information present on both min-tree and maxtree. Moreover, the self-dual connected operators $\rho^{P}$ that are computed on the tree of shapes produce a greater simplification of the image with respect to non dual filters, since they operate simultaneously on the bright and dark components. An example of an attribute filter computed on the tree of shapes representation with the increasing attribute area is shown in Fig. 4. The self-dual operator $\rho^{P}$ is able to remove directly both bright and dark small structures [see the difference image $f-\rho^{P}(f)$ Fig. 4(c)], and it leads to a complete simplification of the image [see the filtered image $\rho^{\mathrm{P}}(f)$ Fig. 4(b)].

\section{Non increasing attributes}

As already explained in the previous section, in order to handle the non increasing attributes, pruning and non pruning strategies have been proposed. The decision about the most suitable strategy depends mainly on the application (e.g., image filtering or decomposition). An intuitive requirement when decomposing an image $f$ based on shape rather than size (e.g., moment of inertia) is that the difference between the image $f$ and the filtered image $\rho^{P}(f)$ is an image which should only contain structures that do not meet the $P$ [26] (i.e., the regions which have been filtered out). It was shown that the pruning strategies can not satisfy this requirement. For example, in the branch of the tree of shapes shown in Fig. $5(a)$ :

$$
(A) N_{5} \longrightarrow \text { (D) } N_{0} \longrightarrow(I) N_{5},
$$

which means that only the node $\mathrm{D}$ has to be removed (i.e., it does not satisfy $P$ ). In this case, there are no pruning strategies that can simultaneously retain A and I, while removing D (e.g., Fig. 5(c) min removes all the nodes A-D-I, while Fig. 5(d) 


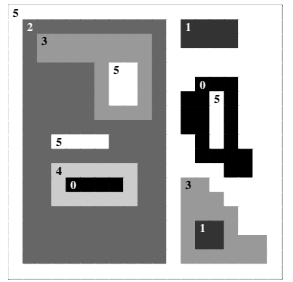

(a)

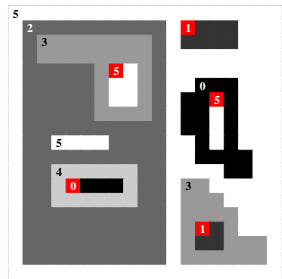

(b)

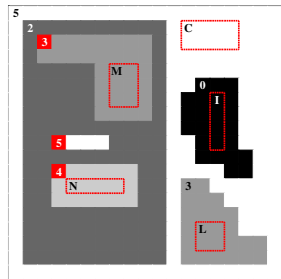

(c)

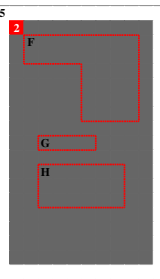

(d)
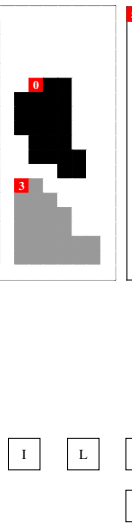

(i)

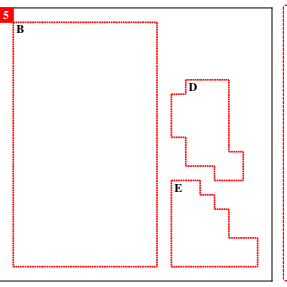

(e)

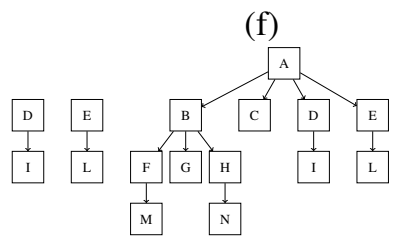

(j)

(k)

Fig. 3: Tree of shapes construction of the grayscale image $f$ (a) with intensities ranging from 0 to 5 . We start by considering the regional extrema (b), then we saturate the components until single flat regions are obtained (c) and associate a node of the a tree to each region $(\mathrm{h})$. Then we iterate until only a single flat region is reached (f). The image is represented as a hierarchical structure $(\mathrm{k})$.

max preserves the whole branch). Furthermore, the authors in [26] proved that also the non pruning strategies such as direct may not perform efficiently. For example, the difference image shown in Fig. 5(e) contains regions which satisfy $P$ (e.g., the nodes F-G-I-N).

The authors in [27] proposed a new strategy for non increasing attributes called subtractive rule, in order to address the previous issues. However, its definition was formulated for the max-tree structure for which the inclusion of the nodes is driven by graylevels. For instance, the definition of subtractive

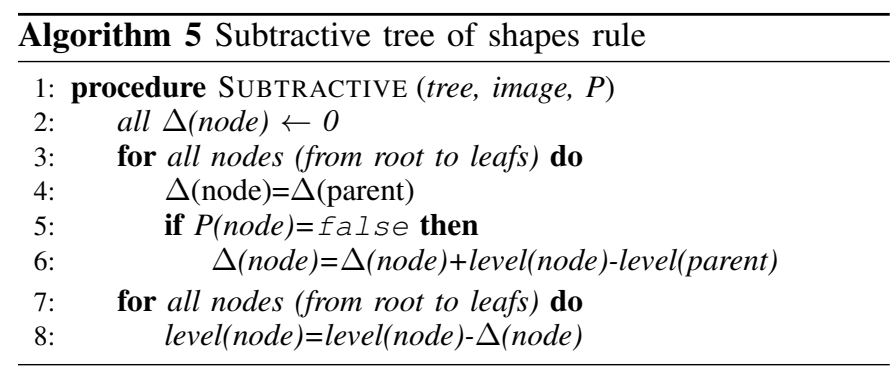

can not be directly applied to the tree of shapes. Particularly, in Algorithm 4 the updating process is achieved by considering all the graylevels in turn starting from the minimum (i.e., max-tree). This approach would make no sense in the tree of shapes structure since the hierarchy between the nodes follows the inclusion relationship of the regions. We propose here a different approach, where the intensity of each descendant node is lowered by the same amount of which the deleted node was lowered. The proposed subtractive function (Algorithm 5) begins with a loop where, for each node, starting from the leafs, a $\Delta$ value is stored. The $\Delta$ is equal to the $\Delta$ of its parent. If the $P$ of the node is false, a new value is added to $\Delta$, equals to the difference between the level of the node and the level of its parent. Finally, for each node, $\Delta$ is used to lower all the sub-components level of the surviving descendants nodes.

In this scenario, for the branch of the tree of shapes previously considered, the attribute filter removes the nodes $\mathrm{D}$ and updates the graylevel of the surviving descendant I.
The filter first computes $\Delta$ and then it updates the level of the node I as follows:

$$
\begin{gathered}
\Delta=(A) N_{5}-(D) N_{0}=5-0=5 \\
(I) N=(I) N_{5}-\Delta=5-(-5)=10
\end{gathered}
$$

The updating process solves the problem found in direct, where regions that satisfy $P$ are lost in the filtered image since the contrast with the local background is not maintained. For example the surviving regions $\mathrm{I}$ and $\mathrm{N}$ do not appear in the filtered image with the direct rule in Fig. 5(e) while they are preserved by the subtractive rule in Fig. 5(f). This effect may become critical when filtering images representing objects in a real scene (i.e., remotely sensed images). For instance, the number of connected components within a filtered image with the direct rule can be much lower than the one contained in an image filtered with subtractive, which means that a part of the information related to the objects is lost. Finally, the updating of the graylevels of the descendants over the tree of shapes introduces new graylevels in the filtered image in Fig. 5(f), which were not present in the original image in Fig. 5(b).

\section{CLASSIFICATION WITH MORPHOLOGICAL ATTRIBUTE PROFILES}

Attribute profiles (APs) were introduced in remote sensing in [39] as a sequential application of attribute filters based on a min-tree (i.e., attribute thickening operation $\phi^{T}$ ) and max-tree (i.e., attribute thinning operation $\gamma^{T}$ ). The AP is obtained by filtering an image $f$ with attribute operators using a predicate with increasing threshold values $\left\{\lambda_{k}\right\}_{L}^{1}$ :

$A P(f)=\left\{\phi^{T_{\lambda_{L}}}(f), \phi^{T_{\lambda_{L-1}}}(f), \ldots, f, \ldots, \gamma^{T_{\lambda_{L-1}}}(u), \gamma^{T_{\lambda_{L}}}(f)\right\}$

with $\phi$ and $\gamma$ being the thickening and thinning operators based on the predicate $T$, respectively, and $T_{\lambda}$ a set of $L$ ordered 
predicates.

APs provide a multilevel characterization of the spatial features which can be useful for the classification of very high resolution remote sensing images [29].

The Self-Dual Attribute Profiles (SDAPs) [29], were proposed as a version of the APs based on self-dual connected operators $\rho^{T}$ computed on the tree of shapes instead of considering a min-tree or max-tree. The use of the tree of shapes as a structure representing the image allows simultaneously to access the information present on the component trees. Moreover, the self-dual connected operators that are computed on the tree of shapes produce a greater simplification of the image with respect to non dual filters, since they operate simultaneously on bright and dark components of the image. SDAPs is obtained by filtering an image $f$ with attribute operators using a predicate with increasing threshold values:

$$
S D A P(f)=\left\{f, \rho^{T_{\lambda_{1}}}(f), \ldots, \rho^{T_{\lambda_{L-1}}}(f), \rho^{T_{\lambda_{L}}}(f)\right\}
$$

with $\rho$ being the self-dual operator based on the predicate $T$, and $T_{\lambda}$ a set of $L$ ordered predicates.

Dalla Mura et al. in [40] proposed Extended Attribute Profiles (EAPs) as the application of APs to hyperspectral data. An EAP is obtained by concatenating the APs (i.e., based on a single attribute) built on several feature components (FCs) extracted by a reduction technique (i.e., PCA) computed on the hyperspectral image. Thus, the EAP can be formally defined as:

$$
E A P=\left\{A P\left(F C_{1}\right), A P\left(F C_{2}\right), \ldots, A P\left(F C_{N}\right)\right\}
$$

Analogously to the definition of EAP, Extended Self-Dual Attribute Profiles (ESDAPs) were proposed in [41]. They are generated by concatenating the SDAPs computed on different components. Each SDAP is built on one of the $N$ features components extracted by a feature reduction transformation from a hyperspectral image:

$E S D A P=\left\{S D A P\left(F C_{1}\right), S D A P\left(F C_{2}\right), \ldots, S D A P\left(F C_{N}\right)\right\}$

In contrast to APs, the SDAPs are composed of $N+1$ images while APs, built with the same sequence of predicates are made up of $2 N+1$ images.

\section{EXPERIMENTAL RESULTS}

The filtering strategies introduced in the previous section are highly relevant in any problem related with the identification of objects of different shape and structure on different scales. In this work we will illustrate their performance on the classification of remotely sensed images. Moreover, we provide the experimental results obtained by classifying stacks of filtered images generated by $\min , \max$, direct and subtractive filter rules applied over the tree of shapes representation (i.e., SDAP). We study the capability of those rules in extracting spatial information from a scene by considering different attributes. Additionally, we compare the performance of those rules in terms of classification accuracy by comparing their application to the min-tree and max-tree (i.e., AP).

\section{A. Data set description and experimental setup}

The first dataset used in our experiments is an image of Rome, Italy, acquired by the QuickBird satellite. The dataset consists of a low-resolution $(2.4 \mathrm{~m})$ multispectral image with four bands Red, Green, Blue and Near Infrared and a high spatial resolution panchromatic image of $0.6 \mathrm{~m}$ resolution. Fig. 6(a) shows the true-colour image while Fig. 6(b) shows the groundtruth data with the 9 classes available. The second dataset is an image of Reykjavik, Iceland, acquired by the IKONOS Earth imaging satellite. As with the other dataset, it consists of a low-resolution (4m) multispectral image with the four bands and a high spatial resolution panchromatic image of $1 \mathrm{~m}$ resolution. A groundtruth dataset of 6 classes reported in Fig. 7(b) is available. For each data set the panchromatic and multispectral images are pansharpened using the undecimated discrete wavelet transform (UDWT) method [42], and the obtained high-resolution multispectral images are used for the classification. For the experiments, the names of the different features used for the classification process will be referred hereinafter as follows:

1) PAN: panchromatic image.

2) $A P$ : attribute profile built from the panchromatic image by using a specific attribute and filter rule.

3) SDAP: self-dual attribute profile built from the panchromatic image by using a specific attribute and filter rule.

4) $M S+A P$ : stacked vector consisting of multispectral images (Red, Green, Blue and Infrared) and AP.

5) $M S+S D A P$ : stacked vector consisting of MS and SDAP.

The attributes and the corresponding threshold values used for building APs and SDAPs are reported in Table. I(a) and Table I(b) for the Rome and Reykjavik data set respectively. The APs are computed by using the implementation of the min-tree and max-tree included in the C++ Milena library [43], while the SDAPs from an adaptation of the code for the tree of shapes provided in the MegaWave2 toolbox [44]. The number of trees of the RF classifier is 200, and all the other options are set with the default values. For each attribute, the table shows the classification result by considering different filtering rules and distinct features configuration. For example, each column (filter rule) of Table IV(a) consists of four different features configuration: AP (21 features), SDAP (11 features), MS (4 features) + AP (21 features) and MS (4 features) + SDAP (11 features). The classification experiments are repeated ten times, randomly selecting $10 \%$ of the references samples as training set, and the mean values of the overall (OA), average (AA) accuracy and kappa $(\mathrm{K})$ coefficient are given.

\section{B. Results}

In the following subsections, we discuss the experimental results obtained for each attribute. Each table reports the classification accuracies for the features AP, SDAP, MS+AP and MS+SDAP. It is taken for granted that every time spectral features MS are added to the classifier, the resulting accuracies can only improve. 


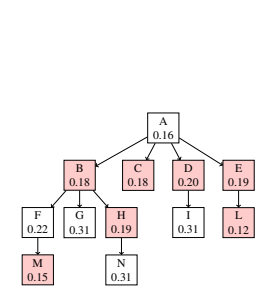

(a)

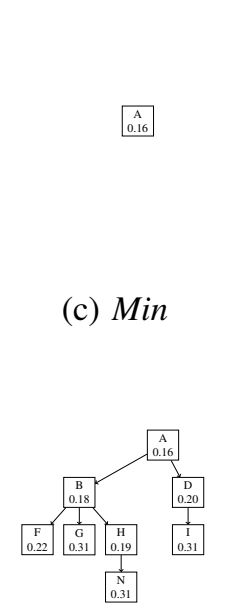

(d) Max

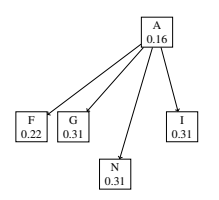

(e) Direct

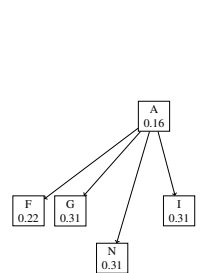

(f) Subtractive

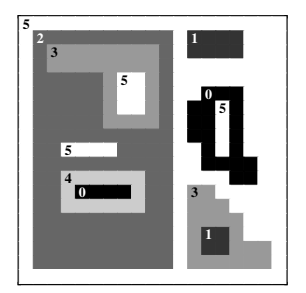

(b)
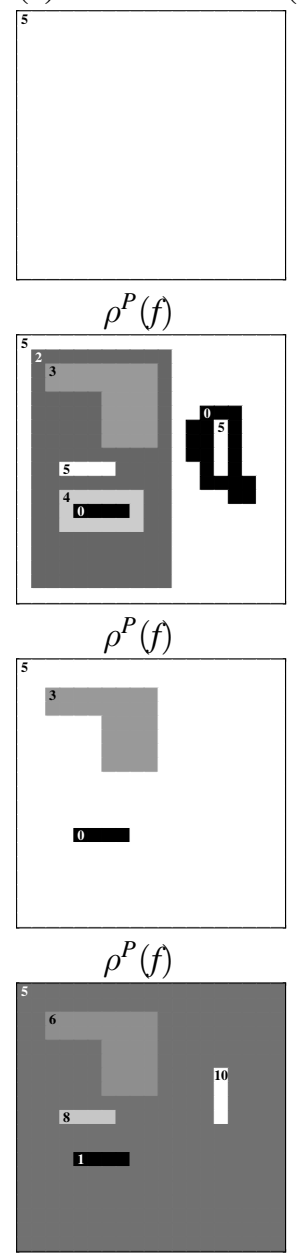

$\rho^{P}(f)$
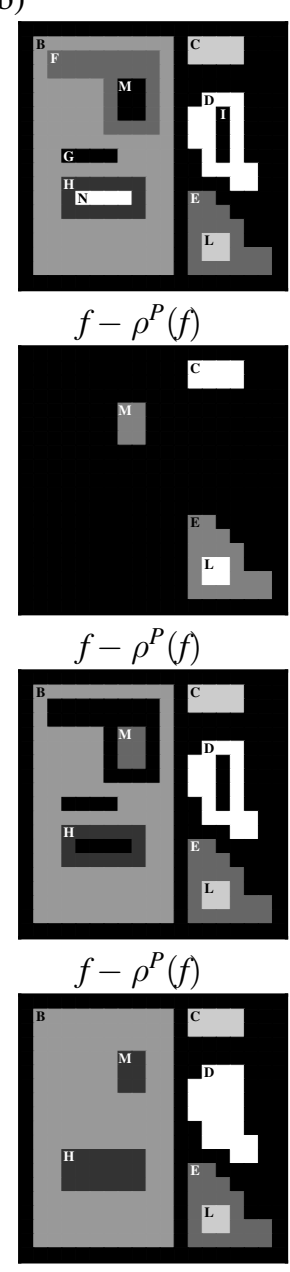

$f-\rho^{P}(f)$
Fig. 5: Attribute filtering of the grayscale image $f(b)$, represented by the tree in (a), using moment of inertia with $P=\alpha(C C) \leq 0.22$ using pruning $(\min (\mathrm{c})$ and $\max (\mathrm{d}))$ and non pruning filtering strategies (direct (e) and subtractive (f)). Each column represents, from left to right, pruned tree, filtered image $\rho^{P}(f)$ and difference image $f-\rho^{P}(f)$.

\begin{tabular}{cc}
\hline Attribute & Thresholds \\
\hline $\begin{array}{c}\text { Area } \\
\text { Standard Deviation } \\
\text { Moment of Inertia }\end{array}$ & $25,100,500,1000,5000,10000,20000,50000,100000,150000$ \\
$5,10,15,20,25,30,35,40,45,50$ \\
\hline \\
Attribute & (a) \\
\hline Area & Thresholds \\
\hline $\begin{array}{c}\text { Standard Deviation } \\
\text { Moment of Inertia }\end{array}$ & $0.20,0.25,0.30,0.35,0.40,0.45,0.50,0.55,0.60,0.65$ \\
\hline & $2.5,0.30,0.35,10,15,20,25,30,35,40$ \\
\hline & (b) \\
& +++
\end{tabular}

TABLE I: Attribute threshold values for the profiles: (a) Rome and (b) Reykjavik data set.

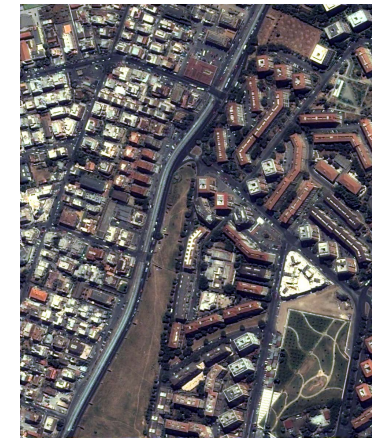

(a)

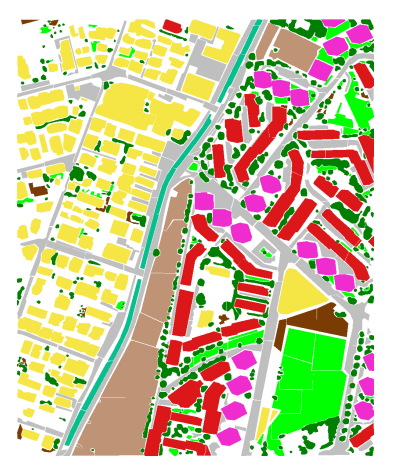

(b)
Thematic classes:

$\square$ Building $\square$ Block $\square$ Road
$\square$ Light Train $\square$ Vegetation $\square$ Trees
$\square$ Bare Soil $\square$ Soil $\quad \square$ Tower

Fig. 6: QuickBird Rome dataset: (a) true color image and (b) groundtruth data.

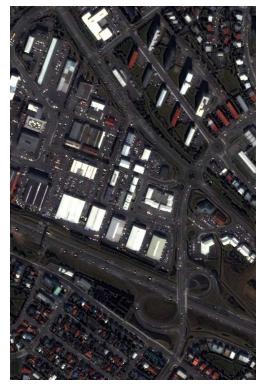

(a)

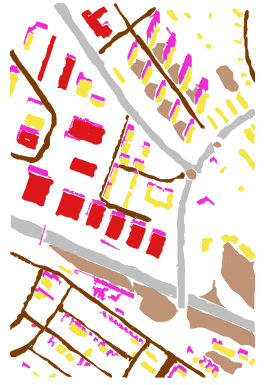

(b)
Thematic classes:

Small building $\square$ Open area $\square$ Shadow

Large building $\square$ Large road $\square$ Street

Fig. 7: IKONOS Reykjavik dataset: (a) true color image and (b) groundtruth data.

1) Area: it belongs to the class of increasing attributes, thus each filter rule generate identical filtered images. In Table III we report the classification results for three feature stack compositions. The first column considers the features PAN and MS+PAN (i.e. the results are reported only for comparison purposes). The second and third columns report AP and SDAP respectively, which are built with the area attribute. When APs and SDAPs are classified, all the classes are detected with a higher precision since they carry a significant amount of geometrical information. The accuracies obtained by the SDAPs are slightly better than those achieved with the APs. In [29] it was already shown the effectiveness of SDAP based on the area attribute for the classification of a very high geometrical resolution scene.

2) Standard deviation: filters based on the non increasing attribute standard deviation perform a multilevel decomposition of the objects in the scene. Specifically, the simplification process is not related to the geometry of 


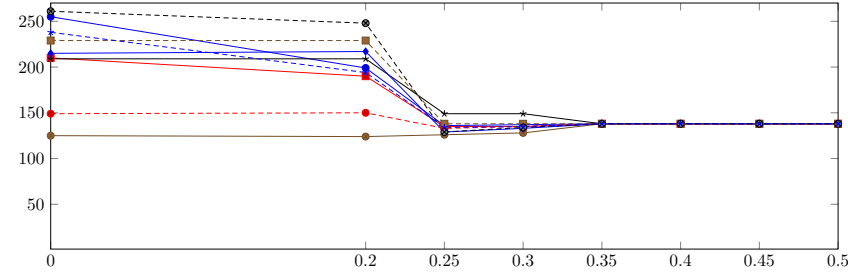

(a)

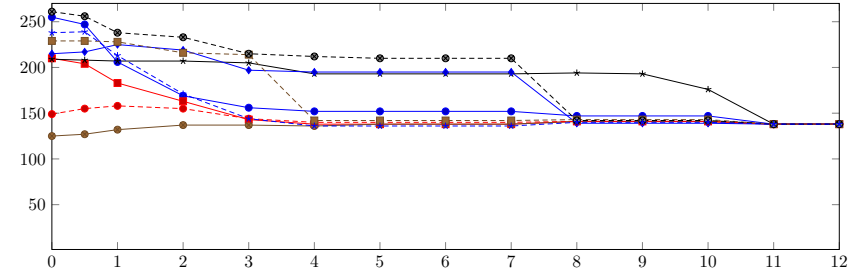

(b)

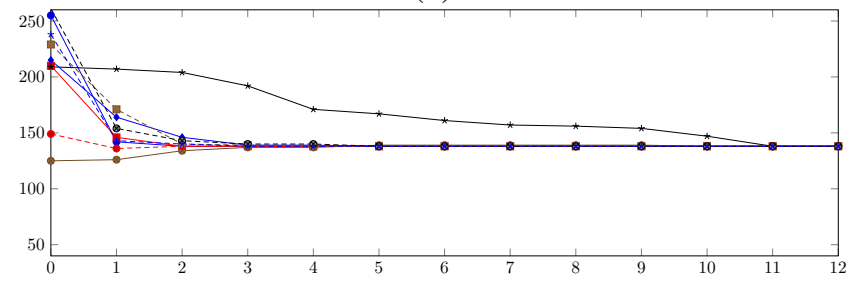

(c)

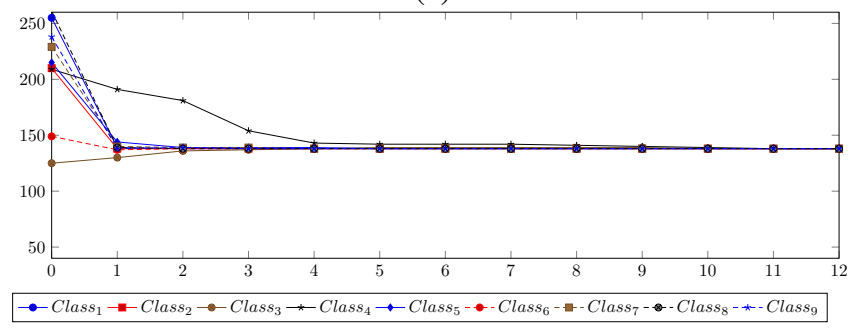

(d)

Fig. 8: The averages of the gray levels of the pixels within each class for each threshold of the moment of inertia attribute in the SDAPs generated by different filter rules (Rome data set): (a) $\min$, (b) max, (c) direct, (d) subtractive.

the shapes but to the modeling of the homogeneity of the graylevels of the pixels belonging to the regions. We start the analysis by looking at the classification accuracies shown in Table IV and notice that the results for the different filter rules are comparable. Even if the standard deviation is a non increasing attribute, all the nodes belonging to a single branch of the tree may or not satisfy the predicate. This is because the attribute does not have a strong non increasing behavior. As a result, a filter which uses pruning and non pruning strategies will generate similar filtered images, and the profiles (i.e., APs or SDAPs) built on different rules will be very similar to each other.

3) Moment of Inertia: filters based on the non increasing attribute moment of inertia are able to discriminate the shape of different structures, since the attribute provides a measure related to the elongation of a region. Contrary to the standard deviation, the classification accuracies obtained with the different filter rules vary greatly to each other, as shown in Table V. This is due to the considerable non increasing trend

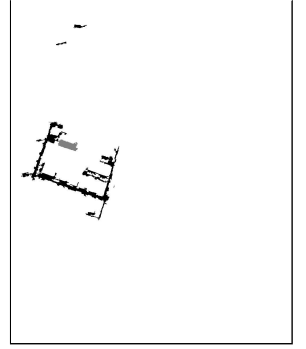

(a)

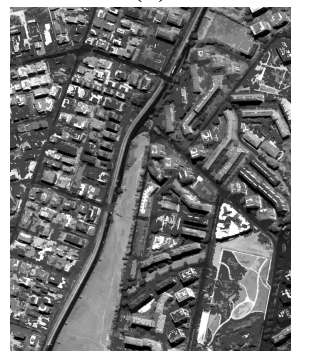

(c)

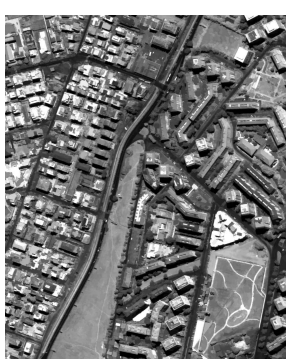

(b)

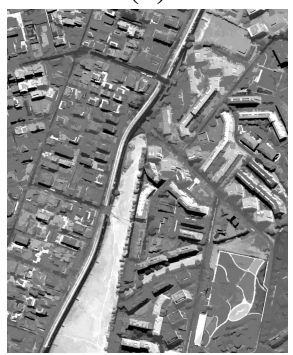

(d)
Fig. 9: Result after filtering the Rome panchromatic image by considering moment of inertia and 0.35 as threshold value. The four filter rules are applied over tree of shapes: (a) $\mathrm{min}$ filtered image, (c) max filtered image, (d) direct filtered image and (e) subtractive filtered image.

along the tree branches of the attribute values, which lead each filter rule to decompose an image in a different way.

We consider each filter rule separately and we provide a detailed analysis for the attribute filters applied over the tree of shapes for the Rome data set (there is an exhaustive literature related to the use of pruning and non pruning strategies with min-tree and max-tree [27]). The graphs in Fig. 8 show the average values of the graylevels of the pixels within each class for each threshold of the moment of inertia attribute in the SDAPs generated by different filter rules. This is useful for understanding the response of each class through the different threshold values of the profile.

When considering the min rule, the filter produces a considerable simplification of the scene. Moreover, the presence of a node that does not fulfill the criterion close to the root of the tree produces the removal of entire branches. For example, the filter starts to remove almost all the regions at the threshold value $\lambda=0.35$ as shown in Fig. 9(a). The plot of Fig. 8(a) indicates that the pixels belonging to the different classes get the same constant value for threshold values greater than $\lambda=0.35$. The images within the profile which are filtered at those thresholds, do not carry any spatial information (i.e., no regions have survived), leading the classifier to achieve poor classification results in both datasets, as shown in Table V.

In the case of max rule, the filter might not perform any effective simplification of the scene leading to filtered images that can be similar to the original image for most part of the threshold set. The plot of Fig. 8(b), shows that the pixels within the classes maintain almost the same grayvalue for all the thresholds. For instance, the filtered image at the threshold value $\lambda=0.35$ shown in Fig. 9(b) is not decomposed yet. As a consequence, the classification accuracies obtained with AP 
and SDAP are similar, since the operator does not perform an effective filtering for the different thresholds (i.e., no structures are extracted).

Different considerations have to be done when the non pruning rules direct and subtractive are taken into account. As shown in Fig. 8(c) and 8(d) the pixel values of the different classes do not take the same constant value at a certain threshold as in the case of $\min$ rule. Moreover, contrary to the max rule, the change in the trend of each class is more visible along the thresholds. This is due to the spatial information represented by the surviving regions after the filtering with non pruning strategies. Looking at the classification results in Table V, SDAPs achieve greater classification results with the subtractive if compared with the direct rule. As discussed in section III, unlike the direct rule, when a node in the tree is deleted, the subtractive propagates new values to its surviving descendants. Consequently, the contrast grayvalue between regions that are not filtered out and the local background is preserved. An example of a subscene of the Rome data set filtered with direct and subtractive is shown in Fig. 10. The effect of the propagation can be see in Table II, which reports the number of connected components within the filtered images with direct and subtractive rules at different threshold values. For each threshold value, the number of connected components after filtering with subtractive is always greater than the one obtained with direct. Regions that satisfy $\mathrm{P}$ may be lost in the filtered image with direct since the contrast with the local background is not maintained. Thus, the classifier can benefit from the spatial information brought by those additional regions. Subtractive can achieve better accuracy results as reported in Table $\mathrm{V}$ and provide more accurate classification maps as shown in Fig. 11. The difference between the considered filter rules in terms of CPU processing time is not relevant. The profiles AP and SDAP are computed in a few seconds only by the rules for both data sets on a computer having Intel(R) Core (TM) i7-4710HQ CPU $2.50 \mathrm{GHz}$ and $16 \mathrm{~GB}$ of memory.

\begin{tabular}{ccccccccccc}
\hline threshold & 0.20 & 0.25 & 0.30 & 0.35 & 0.40 & 0.45 & 0.50 & 0.55 & 0.60 & 0.65 \\
\hline $\begin{array}{c}\text { direct } \\
\text { subtractive }\end{array}$ & 733759 & 559334 & 432206 & 330832 & 264216 & 214168 & 181111 & 155237 & 132170 & 114753 \\
\hline difference & 142 & 376710 & 432749 & 331511 & 264772 & 214608 & 181477 & 155634 & 132531 & 115121 \\
\hline
\end{tabular}

TABLE II: The number of connected components within the filtered images with direct and subtractive rules and their difference number $|C C|_{\text {sub }}-|C C|_{\text {direct }}$ for each threshold values of moment of inertia.

\section{CONCLUSIONS AND FUTURE LINES}

Very high resolution remotely sensed imagery provides precise geometrical information. In this paper, mathematical morphology has been exploited for designing new operators able to filter hierarchical structures which represent an image. In this work, we considered the definition of the filter rules $d i$ rect, max, min and subtractive for the computation of attribute filters over the tree of shapes representation. We generated a tree-based representation of the image, then filtered the

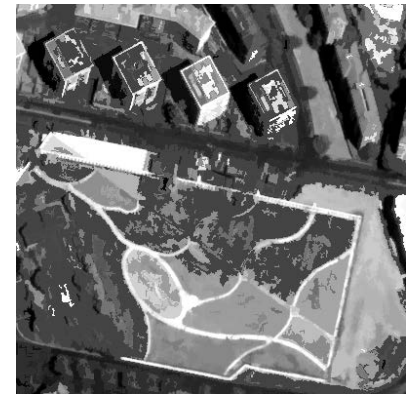

(a)

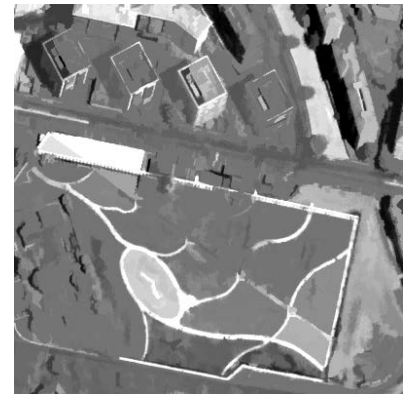

(b)
Fig. 10: Comparison of the direct and subtractive rules when filtering of a subscene of the Rome panchromatic image with inclusion tree using moment of inertia $(0.35$ as threshold value).

representation and finally reconstructed the filtered image from the filtered tree. We showed that subtractive rule preserves contrast grayvalue between regions that are not filtered out and introduces new graylevels in the filtered images which were not present in the original image. We studied the performance of the different rules in terms of classification accuracy in the context of APs and SDAPs, by considering the non increasing attributes standard deviation and moment of inertia. We have proved that when the criterion presents a strong non increasing behavior (i.e., moment of inertia), attribute filters provide heterogeneous profiles for the different filtering strategies. In this case, by looking at the classification accuracies obtained in our experiments, one can conclude that subtractive is the most effective filter rule in our context. Contrary to that, we have shown that if the criterion is more similar to an increasing behavior (i.e., standard deviation), the different filter rules provide similar profiles. Finally, due to the properties of the tree of shapes, we have shown that SDAPs outperform APs in terms of classification accuracies. Although the acceptable time for generating the profiles, our future research aims to develop real-time implementations for large scenes on GPUs. We will work on a new algorithm implemented in parallel fashion in order to compute the tree of shapes and the different filter rules.

\section{REFERENCES}

[1] J. Serra, "Morphologie Mathématique et Genèse des Concrétions Carbonatées des Minerais de per de Lorraine," Sedimentology, vol. 10, pp. 183-208, 1968.

[2] G. M. A. Haas and J. Serra, "Morphologie Mathematique et Granulometries en Place," Annales des Mines, vol. 11, pp. 736-753, 1967.

[3] J. Serra, "Stereology and Structuring Elements," Journal of Microscopy, vol. 95, pp. 93-103, 1972.

[4] _ Image Analysis and Mathematical Morphology. London: Academic Press, 1982.

[5] —-, "Image Analysis and Mathematical Morphology. Volume 2: Theoretical Advances. Edited by Jean Serra," Journal of Microscopy, vol. 2, 1988.

[6] P. Soille and M. Pesaresi, "Advances in Mathematical Morphology Applied to Geoscience and Remote Sensing," IEEE Transactions on Geoscience and Remote Sensing, vol. 40, no. 9, pp. 2042-2055, 2002.

[7] J. A. Benediktsson, L. Bruzzone, J. Chanussot, M. Dalla Mura, P. Salembier, and S. Valero, "Hierarchical Analysis of Remote Sensing Data: Morphological Attribute Profiles and Binary Partition Trees," in Lecture 


\begin{tabular}{rrrr}
\hline & PAN $(1)$ & AP $(21)$ & SDAP $(11)$ \\
\hline AA & $26.64(0.19)$ & $85.06(0.12)$ & $\mathbf{8 7 . 3 4}(\mathbf{0 . 1 2})$ \\
OA & $41.66(0.04)$ & $85.58(0.04)$ & $\mathbf{8 8 . 0 4}(\mathbf{0 . 0 4})$ \\
K & $28.15(0.14)$ & $82.86(0.04)$ & $\mathbf{8 5 . 7 9}(\mathbf{0 . 0 5})$ \\
\hline & MS+PAN $(5)$ & AP $(25)$ & SDAP $(15)$ \\
\hline AA & $70.16(0.11)$ & $94.23(0.05)$ & $\mathbf{9 5 . 1 8 ( \mathbf { 0 . 0 7 } )}$ \\
OA & $75.05(0.03)$ & $94.71(0.03)$ & $\mathbf{9 5 . 5 5}(\mathbf{0 . 0 3})$ \\
K & $70.12(0.04)$ & $93.72(0.04)$ & $\mathbf{9 4 . 7 2}(\mathbf{0 . 0 4})$ \\
\hline
\end{tabular}

(a)

\begin{tabular}{rrrr}
\hline & PAN $(1)$ & AP $(21)$ & SDAP $(11)$ \\
\hline AA & $52.82(0.21)$ & $87.62(0.12)$ & $\mathbf{9 0 . 5 5 ( 0 . 0 8 )}$ \\
OA & $51.13(0.14)$ & $87.12(0.13)$ & $\mathbf{9 0 . 3 2}(\mathbf{0 . 0 6})$ \\
K & $40.91(0.21)$ & $84.45(0.16)$ & $\mathbf{8 8 . 3 1 ( 0 . 0 8 )}$ \\
\hline & MS+PAN $(5)$ & MS + AP $(25)$ & MS + SDAP (15) \\
\hline AA & $76.87(0.12)$ & $92.82(0.06)$ & $\mathbf{9 4 . 2 3 ( 0 . 0 8 )}$ \\
OA & $76.74(0.11)$ & $92.78(0.05)$ & $\mathbf{9 4 . 2 6 ( 0 . 0 7 )}$ \\
K & $71.91(0.14)$ & $91.29(0.06)$ & $\mathbf{9 3 . 0 7 ( 0 . 0 9 )}$ \\
\hline
\end{tabular}

(b)

TABLE III: Classification accuracies of (a) Rome and (b) Reykjavik data set (mean value with its standard deviation in brackets) obtained by the panchromatic and spectral features MS and the AP and SDAP based on the attribute area.

\begin{tabular}{|c|c|c|c|c|}
\hline & MIN & MAX & DIR & SUB \\
\hline & \multicolumn{4}{|c|}{$\mathrm{AP}(21)$} \\
\hline $\mathrm{AA}$ & $76.82(0.09)$ & $74.05(0.09)$ & $75.25(0.07)$ & $76.73(0.09)$ \\
\hline OA & $77.36(0.05)$ & $75.64(0.05)$ & $76.10(0.04)$ & $77.37(0.06)$ \\
\hline \multirow[t]{2}{*}{$\mathrm{K}$} & $72.89(0.07)$ & $70.81(0.07)$ & $71.35(0.05)$ & $72.91(0.08)$ \\
\hline & \multicolumn{4}{|c|}{ SDAP (11) } \\
\hline $\mathrm{AA}$ & $79.83(0.09)$ & $69.53(0.22)$ & $77.46(0.08)$ & $79.21(0.09)$ \\
\hline $\mathrm{OA}$ & $80.74(0.04)$ & $74.22(0.08)$ & $78.82(0.05)$ & $80.13(0.06)$ \\
\hline \multirow[t]{2}{*}{$\mathrm{K}$} & $76.95(0.05)$ & $69.01(0.11)$ & $74.59(0.05)$ & $76.22(0.07)$ \\
\hline & \multicolumn{4}{|c|}{$\mathrm{MS}+\mathrm{AP}(25)$} \\
\hline AA & $88.58(0.06)$ & $86.31(0.07)$ & $87.56(0.06)$ & $88.35(0.07)$ \\
\hline $\mathrm{OA}$ & $89.65(0.04)$ & $88.12(0.04)$ & $88.89(0.05)$ & $89.58(0.04)$ \\
\hline \multirow[t]{2}{*}{$\mathrm{K}$} & $87.68(0.05)$ & $85.85(0.05)$ & $86.76(0.06)$ & $87.59(0.05)$ \\
\hline & \multicolumn{4}{|c|}{$\mathrm{MS}+\mathrm{SDAP}(15)$} \\
\hline $\mathrm{AA}$ & $89.54(0.06)$ & $84.33(0.11)$ & $87.81(0.11)$ & $88.75(0.08)$ \\
\hline $\mathrm{OA}$ & $90.80(0.02)$ & $87.16(0.05)$ & $89.52(0.04)$ & $90.23(0.03)$ \\
\hline $\mathrm{K}$ & $89.05(0.03)$ & $84.69(0.06)$ & $87.51(0.05)$ & $88.37(0.04)$ \\
\hline
\end{tabular}

(a)

\begin{tabular}{|c|c|c|c|c|}
\hline & MIN & MAX & DIR & SUB \\
\hline & \multicolumn{4}{|c|}{$\mathrm{AP}(21)$} \\
\hline AA & $78.57(0.35)$ & $85.20(0.16)$ & $81.92(0.29)$ & $82.12(0.21)$ \\
\hline $\mathrm{OA}$ & $77.96(0.43)$ & $84.62(0.14)$ & $81.26(0.33)$ & $81.50(0.24)$ \\
\hline \multirow[t]{2}{*}{$\mathrm{K}$} & $73.37(0.51)$ & $81.43(0.17)$ & $77.37(0.39)$ & $77.66(0.29)$ \\
\hline & \multicolumn{4}{|c|}{ SDAP (11) } \\
\hline AA & $85.96(0.12)$ & $84.86(0.16)$ & $86.13(0.13)$ & $87.32(0.17)$ \\
\hline $\mathrm{OA}$ & $85.64(0.11)$ & $84.12(0.17)$ & $85.76(0.12)$ & $86.90(0.15)$ \\
\hline \multirow[t]{2}{*}{$\mathrm{K}$} & $82.64(0.13)$ & $80.83(0.21)$ & $82.79(0.14)$ & $84.17(0.19)$ \\
\hline & \multicolumn{4}{|c|}{$\mathrm{MS}+\mathrm{AP}(25)$} \\
\hline $\mathrm{AA}$ & $88.87(0.12)$ & $91.45(0.08)$ & $89.62(0.08)$ & $89.65(0.11)$ \\
\hline $\mathrm{OA}$ & $88.95(0.12)$ & $91.40(0.08)$ & $89.64(0.07)$ & $89.67(0.11)$ \\
\hline \multirow[t]{2}{*}{$\mathrm{K}$} & $86.66(0.15)$ & $89.62(0.09)$ & $87.49(0.09)$ & $87.53(0.12)$ \\
\hline & \multicolumn{4}{|c|}{ MS + SDAP (15) } \\
\hline $\mathrm{AA}$ & $91.77(0.11)$ & $90.71(0.04)$ & $91.38(0.09)$ & $91.98(0.07)$ \\
\hline $\mathrm{OA}$ & $91.72(0.09)$ & $90.64(0.03)$ & $91.33(0.09)$ & $91.91(0.07)$ \\
\hline $\mathrm{K}$ & $90.01(0.11)$ & $88.70(0.04)$ & $89.54(0.11)$ & $90.24(0.08)$ \\
\hline
\end{tabular}

(b)

TABLE IV: Classification accuracies of (a) Rome and (b) Reykjavik data set (mean value with its standard deviation in bracket) for each filter rule. First. the spatial features APs and SDAPs are considered (non increasing attribute standard deviation). Finally. the spectral information MS is added as additional features. The number of features is reported in the parentheses.

\begin{tabular}{|c|c|c|c|c|}
\hline & MIN & MAX & DIR & SUB \\
\hline & \multicolumn{4}{|c|}{ AP (21) } \\
\hline AA & $18.60(0.01)$ & $69.11(0.19)$ & $68.04(0.21)$ & $82.07(0.14)$ \\
\hline $\mathrm{OA}$ & $37.10(0.01)$ & $75.78(0.07)$ & $74.36(0.09)$ & $83.08(0.07)$ \\
\hline \multirow[t]{2}{*}{$\mathrm{K}$} & $18.87(0.01)$ & $71.15(0.08)$ & $69.40(0.11)$ & $79.85(0.08)$ \\
\hline & \multicolumn{4}{|c|}{ SDAP (11) } \\
\hline AA & $43.60(0.37)$ & $51.96(0.17)$ & $69.05(0.28)$ & $83.85(0.21)$ \\
\hline $\mathrm{OA}$ & $57.87(0.44)$ & $62.17(0.04)$ & $74.66(0.11)$ & $84.50(0.07)$ \\
\hline \multirow[t]{2}{*}{$\mathrm{K}$} & $48.95(0.58)$ & $54.40(0.05)$ & $69.79(0.14)$ & $81.56(0.08)$ \\
\hline & \multicolumn{4}{|c|}{$\mathrm{MS}+\mathrm{AP}(25)$} \\
\hline AA & $38.48(0.69)$ & $88.62(0.13)$ & $88.35(0.16)$ & $92.20(0.07)$ \\
\hline $\mathrm{OA}$ & $56.58(0.65)$ & $90.25(0.05)$ & $89.91(0.08)$ & $92.76(0.05)$ \\
\hline \multirow[t]{2}{*}{ K } & $46.10(0.89)$ & $88.41(0.06)$ & $88.00(0.09)$ & $91.40(0.06)$ \\
\hline & \multicolumn{4}{|c|}{ MS + SDAP (15) } \\
\hline AA & $76.40(0.64)$ & $78.03(0.08)$ & $87.66(0.13)$ & $92.54(0.11)$ \\
\hline $\mathrm{OA}$ & $82.07(0.36)$ & $81.78(0.04)$ & $89.25(0.04)$ & $92.93(0.06)$ \\
\hline $\mathrm{K}$ & $78.48(0.45)$ & $78.23(0.05)$ & $87.22(0.05)$ & $91.60(0.08)$ \\
\hline
\end{tabular}

(a)

\begin{tabular}{|c|c|c|c|c|}
\hline & MIN & MAX & DIR & SUB \\
\hline & \multicolumn{4}{|c|}{ AP (21) } \\
\hline AA & $31.72(0.01)$ & $76.84(0.11)$ & $79.37(0.06)$ & $84.08(0.08)$ \\
\hline $\mathrm{OA}$ & $32.10(0.01)$ & $75.63(0.12)$ & $78.51(0.07)$ & $83.38(0.09)$ \\
\hline \multirow[t]{2}{*}{$\mathrm{K}$} & $15.18(0.02)$ & $70.57(0.14)$ & $74.04(0.08)$ & $79.93(0.11)$ \\
\hline & \multicolumn{4}{|c|}{ SDAP (11) } \\
\hline AA & $73.95(0.38)$ & $69.25(0.12)$ & $78.30(0.13)$ & $85.98(0.13)$ \\
\hline $\mathrm{OA}$ & $72.58(0.47)$ & $68.00(0.08)$ & $77.32(0.12)$ & $85.42(0.11)$ \\
\hline \multirow[t]{2}{*}{$\mathrm{K}$} & $66.93(0.54)$ & $61.35(0.11)$ & $72.61(0.15)$ & $82.40(0.13)$ \\
\hline & \multicolumn{4}{|c|}{$\mathrm{MS}+\mathrm{AP}(25)$} \\
\hline AA & $59.42(0.71)$ & $85.87(0.06)$ & $87.94(0.11)$ & $90.47(0.07)$ \\
\hline $\mathrm{OA}$ & $60.21(0.67)$ & $85.59(0.05)$ & $87.81(0.09)$ & $90.38(0.06)$ \\
\hline \multirow[t]{2}{*}{$\mathrm{K}$} & $51.53(0.85)$ & $82.60(0.06)$ & $85.29(0.11)$ & $88.39(0.07)$ \\
\hline & \multicolumn{4}{|c|}{ MS + SDAP (15) } \\
\hline AA & $87.58(0.11)$ & $81.58(0.08)$ & $86.71(0.12)$ & $90.64(0.06)$ \\
\hline $\mathrm{OA}$ & $87.50(0.11)$ & $81.47(0.07)$ & $86.57(0.12)$ & $90.57(0.06)$ \\
\hline $\mathrm{K}$ & $84.91(0.13)$ & $77.62(0.09)$ & $83.79(0.15)$ & $88.62(0.07)$ \\
\hline
\end{tabular}

(b)

TABLE V: Classification accuracies of (a) Rome and (b) Reykjavik data set (mean value with its standard deviation in bracket) for each filter rule. First. the spatial features APs and SDAPs are considered (non increasing attribute moment of inertia). Finally. the spectral information MS is added as additional features. The number of features is reported in the parentheses. 


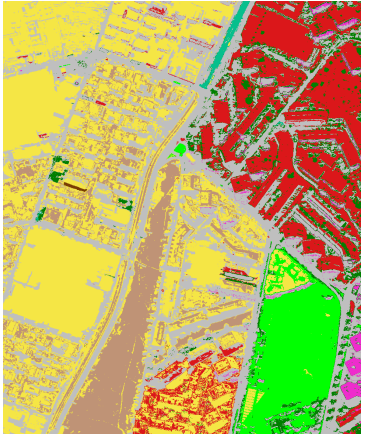

(a)

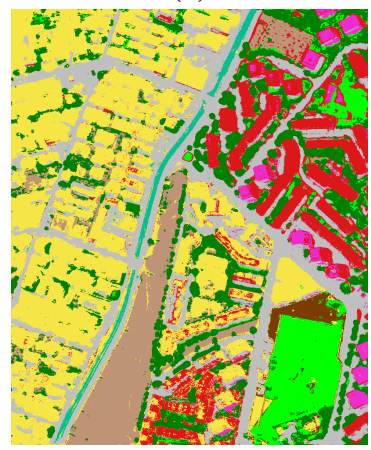

(e)

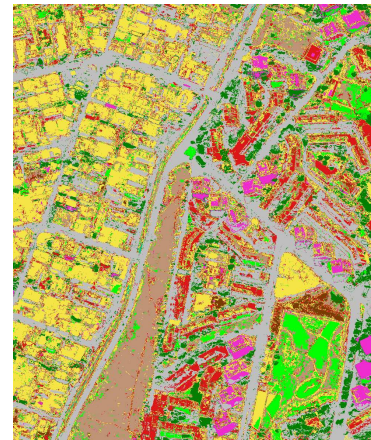

(b)

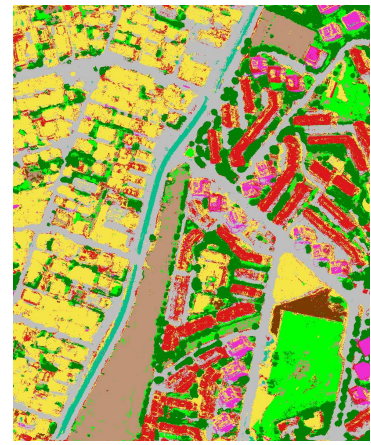

(f)

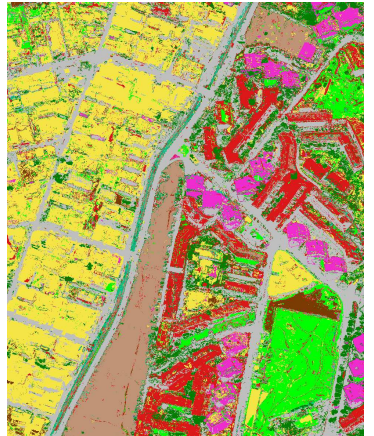

(c)

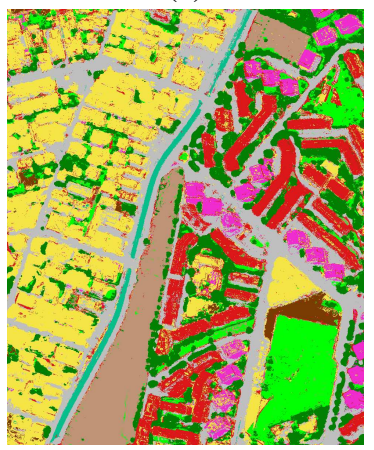

(g)

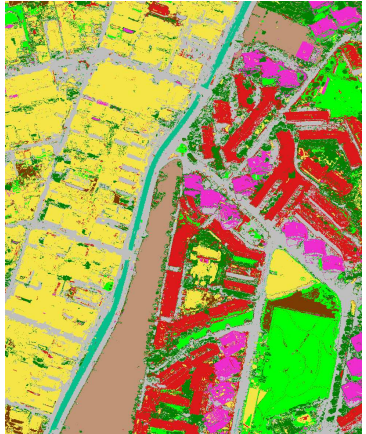

(d)

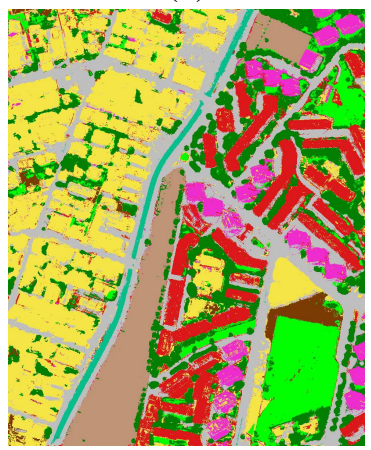

(h)

Fig. 11: Classification maps for the experiments reported in Table V(a) using a single training and test set: (a) SDAP with min, (b) SDAP with $\max$, (c) SDAP with direct, (d) SDAP with subtractive, (e) MS+SDAP with min, (f) MS+SDAP with max, (g) $\mathrm{MS}+\mathrm{SDAP}$ with direct and (h) MS+SDAP with subtractive.

Notes in Computer Science (including subseries Lecture Notes in Artificial Intelligence and Lecture Notes in Bioinformatics), vol. 6671 LNCS, 2011, pp. 306-319.

[8] E. Aptoula and S. Lefèvre, "A Comparative Study on Multivariate Mathematical Morphology," Pattern Recognition, vol. 40, no. 11, pp. 2914-2929, 2007.

[9] A. Meijster and M. H. F. Wilkinson, "A Comparison of Algorithms for Connected Set Openings and Closings," IEEE Transactions on Pattern Analysis and Machine Intelligence, vol. 24, no. 4, pp. 484-494, 2002.

[10] F. Zana and J. C. Klein, "Segmentation of Vessel-Like Patterns Using Mathematical Morphology and Curvature Evaluation," IEEE Transactions on Image Processing, vol. 10, no. 7, pp. 1010-1019, 2001.

[11] E. M. Sigursson, S. Valero, J. A. Benediktsson, J. Chanussot, H. Talbot, and E. Stefánsson, "Automatic Retinal Vessel Extraction Based on Directional Mathematical Morphology and Fuzzy Classification," Pattern Recognition Letters, vol. 47, pp. 164-171, 2014.

[12] P. Soille, Morphological Image Analysis: Principles and Applications, 2nd ed. Springer-Verlag Berlin Heidelberg, 2004.

[13] P. Salembier and J. Serra, "Flat Zones Filtering, Connected Operators, and Filters by Reconstruction," IEEE Transactions on Image Processing, vol. 4, no. 8, pp. 1153-1160, 1995.

[14] P. Salembier and M. Wilkinson, "Connected Operators," IEEE Signal Processing Magazine, vol. 26, no. 6, 2009.

[15] M. Pesaresi and J. A. Benediktsson, "A New Approach for the Morphological Segmentation of High-Resolution Satellite Imagery," IEEE Transactions on Geoscience and Remote Sensing, vol. 39, no. 2, pp. 309-320, 2001.

[16] J. A. Benediktsson, M. Pesaresi, and K. Arnason, "Classification and Feature Extraction for Remote Sensing Images From Urban Areas Based on Morphological Transformations," IEEE Transactions on Geoscience and Remote Sensing, vol. 41, no. 9, pp. 1940-1949, 2003.

[17] E. J. Breen and R. Jones, "Attribute Openings, Thinnings, and Granulometries," Computer Vision and Image Understanding, vol. 64, no. 3, pp. 377-389, nov 1996.

[18] M. H. F. Wilkinson, H. Gao, W. H. Hesselink, J. E. Jonker, and A. Meijster, "Concurrent Computation of Attribute Filters on Shared Memory Parallel Machines," IEEE Transactions on Pattern Analysis and Machine Intelligence, vol. 30, no. 10, pp. 1800-1813, 2008.
[19] Y. Xu, E. Carlinet, T. Geraud, and L. Najman, "Efficient Computation of Attributes and Saliency Maps on Tree-Based Image Representations," in Mathematical Morphology and Its Application to Signal and Image Processing - Proc. of the 12th Intl. Symp. on Mathematical Morphology (ISMM), ser. Lecture Notes in Computer Science, vol. 9082. Springer, 2015, pp. 693-704.

[20] G. K. Ouzounis and M. H. F. Wilkinson, "Mask-Based SecondGeneration Connectivity and Attribute Filters," IEEE Transactions on Pattern Analysis and Machine Intelligence, vol. 29, no. 6, pp. 990-1004, 2007.

[21] P. Salembier, A. Oliveras, and L. Garrido, "Antiextensive Connected Operators for Image and Sequence Processing," IEEE Transactions on Image Processing, vol. 7, no. 4, pp. 555-570, 1998.

[22] P. Salembier, "Connected Operators Based on Region-Trees," in Proceedings of the 15st International Conference on Image Processing (ICIP), 2008, pp. 2176-2179.

[23] P. Monasse and F. Guichard, "Fast Computation of a Contrast-Invariant Image Representation," IEEE Transactions on Image Processing, vol. 9, no. 5 , pp. $860-872,2000$.

[24] H. J. A. M. Heijmans, "Theoretical Aspects of Gray-Level Morphology," IEEE Transactions on Pattern Analysis and Machine Intelligence, vol. 13 , no. 6 , pp. 568-582, 1991.

[25] J. Serra and P. Salembier, "Connected Operators and Pyramids," in Procedings SPIE 2030, Image Algebra and Morphological Image Processing IV, 1993, pp. 65-76.

[26] E. R. Urbach and M. H. F. Wilkinson, "Shape-Only Granulometries and Grey-Scale Shape Filters," in Mathematical Morphology and Its Application to Signal and Image Processing - Proc. of the VIth Intl. Symp. on Mathematical Morphology (ISMM), vol. 6, 2002, pp. 305314.

[27] E. R. Urbach, J. B. T. M. Roerdink, and M. H. F. Wilkinson, "Connected Shape-Size Pattern Spectra for Rotation and Scale-Invariant Classification of Gray-Scale Images," IEEE Transactions on Pattern Analysis and Machine Intelligence, vol. 29, no. 2, pp. 272-285, 2007.

[28] M. Dalla Mura, "Advanced Tecniques Based on Mathematical Morphology for the Analysis of Remote Sensing Images," Ph.D. dissertation, University of Trento and University of Iceland, 2011.

[29] M. Dalla Mura, J. A. Benediktsson, and L. Bruzzone, "Self-dual 
Attribute Profiles for the Analysis of Remote Sensing Images," in Mathematical Morphology and Its Application to Signal and Image Processing - Proc. of the 10h Intl. Symp. on Mathematical Morphology (ISMM), P. Soille, M. Pesaresi, and G. Ouzounis, Eds. Springer Berlin Heidelberg, 2011, pp. 320-330.

[30] G. Cavallaro, M. Dalla Mura, J. A. Benediktsson, and L. Bruzzone, “A Comparison of Self-Dual Attribute Profiles Based on Different Filter Rules for Classification," in Proceedings of the IEEE International Geoscience and Remote Sensing Symposium (IGARSS), 2014, pp. 12651268.

[31] V. Caselles and P. Monasse, Geometric Description of Images as Topographic Maps, 1st ed. Springer-Verlag Berlin Heidelberg, 1984.

[32] G. K. Ouzounis and M. H. F. Wilkinson, "Partition-Induced Connections and Operators for Pattern Analysis," Pattern Recognition, vol. 43, no. 10 pp. 3193-3207, 2010.

[33] G. K. Ouzounis, M. Pesaresi, and P. Soille, "Differential Area Profiles: Decomposition Properties and Efficient Computation," IEEE Transactions on Pattern Analysis and Machine Intelligence, vol. 34, no. 8, pp. 1533-1548, 2012.

[34] E. Carlinet and T. Geraud, "A Comparative Review of Component Tree Computation Algorithms," IEEE Transactions on Image Processing, vol. 23, no. 9, pp. 3885-3895, 2014.

[35] Y. Xu, T. Géraud, and L. Najman, "Morphological Filtering in Shape Spaces: Applications Using Tree-Based Image Representations," 21st International Conference on Pattern Recognition (ICPR 2012), vol. 5, pp. 2-5, 2012. [Online]. Available: http://ieeexplore.ieee.org/xpls/abs_all.jsp?arnumber=6460177

[36] Y. Song, "A Topdown Algorithm for Computation of Level Line Trees," IEEE Transactions on Image Processing, vol. 16, no. 8, pp. 2107-2116, 2007.

[37] T. Géraud, E. Carlinet, S. Crozet, and L. Najman, "A Quasi-Linear Algorithm to Compute the Tree of Shapes of $\mathrm{nD}$ Images," in Lecture Notes in Computer Science (including subseries Lecture Notes in Artificial Intelligence and Lecture Notes in Bioinformatics), vol. 7883 LNCS, 2013, pp. 98-110.

[38] S. Crozet and T. Geraud, "A First Parallel Algorithm to Compute the Morphological Tree of Shapes of $\mathrm{nD}$ Images," in Proceedings of the IEEE International Conference on Image Processing (ICIP), 2014, pp. 2933-2937.

[39] M. Dalla Mura, J. A. Benediktsson, B. Waske, and L. Bruzzone, "Morphological Attribute Profiles for the Analysis of Very High Resolution Images," IEEE Transactions on Geoscience and Remote Sensing, vol. 48, no. 10 , pp. 3747-3762, 2010.

[40] M. Dalla Mura, J. Atli Benediktsson, B. Waske, and L. Bruzzone, "Extended Profiles with Morphological Attribute Filters for the Analysis of Hyperspectral Data," pp. 5975-5991, 2010.

[41] G. Cavallaro, M. Dalla Mura, J. A. Benediktsson, and L. Bruzzone, "Extended Self-Dual Attribute Profiles for the Classification of Hyperspectral Images," IEEE Geoscience and Remote Sensing Letters, vol. 99, pp. 1-5, 2015.

[42] F. Pálsson, J. R. Sveinsson, J. A. Benediktsson, and H. Aanæs, "Classification of Pansharpened Urban Satellite Images," IEEE Journal of Selected Topics in Applied Earth Observations and Remote Sensing, vol. 5, no. 1, pp. 281-297, 2012.

[43] R. Levillain, T. Géraud, and L. Najman, "Milena: Write Generic Morphological Algorithms Once, Run on Many Kinds of Images," in Lecture Notes in Computer Science (including subseries Lecture Notes in Artificial Intelligence and Lecture Notes in Bioinformatics), vol. 5720 LNCS, 2009, pp. 295-306.

[44] M. L. Froment J. and M. J. (1993) Megawave2. [Online]. Available: http://megawave.cmla.ens-cachan.fr/index.php

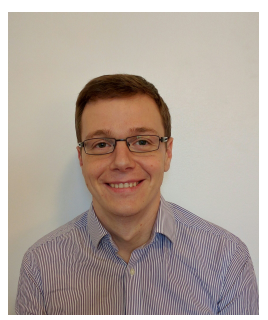

Gabriele Cavallaro received the B.S. and M.S. degrees in telecommunications engineering from the University of Trento, Trento, Italy, in 2011 and 2013, respectively. At present he is a Ph.D. student at the University of Iceland, Reykjavik, Iceland. He was the recipient of the IEEE GRSS Third Prize in the Student Paper Competition of the 2015 IEEE International Geoscience and Remote Sensing Symposium 2015 (Milan, Italy, July 2015). He serves as a reviewer for IEEE Geoscience and Remote Sensing Letters and IEEE Journal of Selected Topics in Earth Observations and Remote Sensing. His research interests include remote sensing and analysis of very high geometrical and spectral resolution images with the current focus on mathematical morphology and high performance computing.

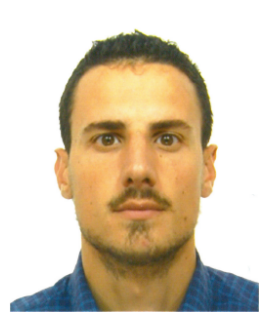

Mauro Dalla Mura Mauro Dalla Mura (S'08 M'11) received the laurea (B.E.) and laurea specialistica (M.E.) degrees in Telecommunication Engineering from the University of Trento, Italy, in 2005 and 2007, respectively. He obtained in 2011 a joint $\mathrm{Ph} . \mathrm{D}$. degree in Information and Communication Technologies (Telecommunications Area) from the University of Trento, Italy and in Electrical and Computer Engineering from the University of Iceland, Iceland. In 2011 he was a Research fellow at Fondazione Bruno Kessler, Trento, Italy, conducting research on computer vision. He is currently an Assistant Professor at Grenoble Institute of Technology (Grenoble INP), France. He is conducting his research at the Grenoble Images Speech Signals and Automatics Laboratory (GIPSA-Lab). His main research activities are in the fields of remote sensing, image processing and pattern recognition. In particular, his interests include mathematical morphology, classification and multivariate data analysis. Dr. Dalla Mura was the recipient of the IEEE GRSS Second Prize in the Student Paper Competition of the 2011 IEEE IGARSS 2011 and co-recipient of the Best Paper Award of the International Journal of Image and Data Fusion for the year 2012-2013 and the Symposium Paper Award for IEEE IGARSS 2014. He is a Reviewer of IEEE Transactions on Geoscience and Remote Sensing, IEEE Geoscience and Remote Sensing Letters, IEEE Journal of Selected Topics in Earth Observations and Remote Sensing, IEEE Journal of Selected Topics in Signal Processing, Pattern Recognition Letters, ISPRS Journal of Photogrammetry and Remote Sensing, Photogrammetric Engineering and Remote Sensing (PERS). He is a member of the Geoscience and Remote Sensing Society (GRSS) and IEEE GRSS Data Fusion Technical Committee (DFTC) and Secretary of the IEEE GRSS French Chapter (2013-2016). He was a lecturer at the RSSS12 - Remote Sensing Summer School 2012 (organized by the IEEE GRSS), Munich, Germany. 


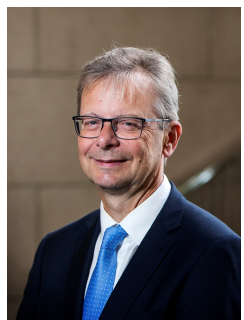

Jón Atli Benediktsson Jón Atli Benediktsson received the Cand.Sci. degree in electrical engineering from the University of Iceland, Reykjavik, in 1984 and the M.S.E.E. and Ph.D. degrees in electrical engineering from Purdue University, West Lafayette, IN, in 1987 and 1990, respectively. On July 1, 2015 he became the Rector of the University of Iceland. From 2009 to 2015 he was the Pro Rector of Science and Academic Affairs and Professor of Electrical and Computer Engineering at the University of Iceland. His research interests are in remote sensing, biomedical analysis of signals, pattern recognition, image processing, and signal processing, and he has published extensively in those fields. Prof Benediktsson was the 2011-2012 President of the IEEE Geoscience and and Remote Sensing Society (GRSS) and has been on the GRSS AdCom since 2000. He was Editor in Chief of the IEEE Transactions on Geoscience and Remote Sensing (TGRS) from 2003 to 2008 and has served as Associate Editor of TGRS since 1999, the IEEE Geoscience and Remote Sensing Letters since 2003 and IEEE Access since 2013. He is on the Editorial Board of the Proceedings of the IEEE, the International Editorial Board of the International Journal of Image and Data Fusion and was the Chairman of the Steering Committee of IEEE Journal of Selected Topics in Applied Earth Observations and Remote Sensing (J-STARS) 2007-2010. Prof. Benediktsson is a cofounder of the biomedical start up company Oxymap (www.oxymap.com). $\mathrm{He}$ is a Fellow of the IEEE and a Fellow of SPIE. Prof. Benediktsson is a member of the 2014 IEEE Fellow Committee. He received the Stevan J. Kristof Award from Purdue University in 1991 as outstanding graduate student in remote sensing. In 1997, Dr. Benediktsson was the recipient of the Icelandic Research Council's Outstanding Young Researcher Award, in 2000, he was granted the IEEE Third Millennium Medal, in 2004, he was a co-recipient of the University of Iceland's Technology Innovation Award, in 2006 he received the yearly research award from the Engineering Research Institute of the University of Iceland, and in 2007, he received the Outstanding Service Award from the IEEE Geoscience and Remote Sensing Society. He was co-recipient of the 2012 IEEE Transactions on Geoscience and Remote Sensing Paper Award and in 2013 he was co-recipient of the IEEE GRSS Highest Impact Paper Award. In 2013 he received the IEEE/VFI Electrical Engineer of the Year Award. In 2014 he was a co-recipient of the International Journal of Image and Data Fusion Best Paper Award. He is a member of the Association of Chartered Engineers in Iceland (VFI), Societas Scinetiarum Islandica and Tau Beta Pi.

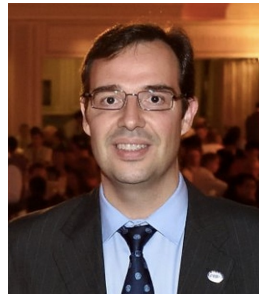

Antonio Plaza (M05-SM07-F15) is the Head of the Hyperspectral Computing Laboratory at the Department of Technology of Computers and Communications, University of Extremadura. His main research interests comprise hyperspectral data processing and parallel computing of remote sensing data. He has authored more than 500 publications, including more than 170 journal papers (more than 120 in IEEE journals), 20 book chapters, and over 250 peer-reviewed conference proceeding papers. $\mathrm{He}$ has guest edited 9 special issues on hyperspectral remote sensing for different journals. Dr. Plaza is a Fellow of IEEE for contributions to hyperspectral data processing and parallel computing of Earth observation data. He is a recipient of the recognition of Best Reviewers of the IEEE Geoscience and Remote Sensing Letters (in 2009) and a recipient of the recognition of Best Reviewers of the IEEE Transactions on Geoscience and Remote Sensing (in 2010), for which he served as Associate Editor in 20072012. He is also an Associate Editor for IEEE Access, and was a member of the Editorial Board of the IEEE Geoscience and Remote Sensing Newsletter (2011-2012) and the IEEE Geoscience and Remote Sensing Magazine (2013). $\mathrm{He}$ was also a member of the steering committee of the IEEE Journal of Selected Topics in Applied Earth Observations and Remote Sensing (JSTARS). He is a recipient of the Best Column Award of the IEEE Signal Processing Magazine in 2015, the 2013 Best Paper Award of the JSTARS journal, and the most highly cited paper (2005-2010) in the Journal of Parallel and Distributed Computing. He received best paper awards at the IEEE International Conference on Space Technology and the IEEE Symposium on Signal Processing and Information Technology. He served as the Director of Education Activities for the IEEE Geoscience and Remote Sensing Society (GRSS) in 2011-2012, and is currently serving as President of the Spanish Chapter of IEEE GRSS. He has reviewed more than 500 manuscripts for over 50 different journals. He is currently serving as the Editor-in-Chief of the IEEE Transactions on Geoscience and Remote Sensing journal. Additional information: http://www.umbc.edu/rssipl/people/aplaza. 